\title{
Effect of Ag Doping on the Electronic Structure and Optical Properties of $\mathrm{ZnO}(0001)$ Surface
}

\author{
Qian Xiang ${ }^{*}$, Shutao Zhao ${ }^{1}$, Yanning $\mathrm{Wu}^{1}$, and Guangdong Liu ${ }^{1}$ \\ ${ }^{1}$ School of Physics and Electronic Engineering, Fuyang Normal College, Fuyang 236037, China
}

\begin{abstract}
Using first-principle calculations, the geometrical structure, the electronic and optical properties of Ag-doped $\mathrm{ZnO}(0001)$ surface have been investigated. We found that $\mathrm{Ag}$-doped $\mathrm{ZnO}(0001)$ surface is more easily formed on the first layer. On the other hand, the doped surface has gradually become an equipotential body, showing obvious metallic characteristics. We found that a new peak appeared in the low energy region after Ag doping, which was mainly due to the electron transition between the two orbital levels of Ag-4d and O-2p.
\end{abstract}

\section{Introduction}

$\mathrm{ZnO}$ is a direct wide band $\mathrm{gap}(3.37 \mathrm{eV})$ semiconductor with a large excitation binding energy of $60 \mathrm{meV}$ at room temperature[1]. Thanks to these properties, $\mathrm{ZnO}$ is used as a photoelectronic material for solar cells, gas sensors, transistors[2-3]. In order to change its electronic and optical properties, the doping of $\mathrm{ZnO}$ surface has been a popular technique, such as $\mathrm{F}-\mathrm{B}[4], \mathrm{Cu}[5], \mathrm{Al}[6]$. Due to the $\mathrm{ZnO}$ crystal has a spontaneous polarization direction along the $\mathrm{c}$ axis,different polar surfaces exhibit different growth characteristics.[79]. Lately, from the experiment, Suat Pat[10] found the $\mathrm{ZnO}$ and AZO thin films have high transparency using RF sputtering technique. Long xing $\mathrm{Su}[11]$ has reported Be doping lead to $\mathrm{ZnO}$ surface gap widening. From the theory, Jianping Xiao[12] has proposed that $\mathrm{Cu}-$ doped $\mathrm{ZnO}(0001)$ surface promote efficient formation of oxygen vacancies. Haifeng Zhang[13] indicated that $\mathrm{Si}$ atoms adsorbed on $\mathrm{ZnO}$ (0001) surface lead to a slightly blue shift phenomenon. Mohammed Ali Lahmer[14] has reported that hydrogen adsorption is more favored on $\mathrm{Mg}$ doping $\mathrm{ZnO}(000 \overline{1})$ surface. So far, Ag-doped $\mathrm{ZnO}(0001)$ surface has few reports on theoretical studies.

In this paper, geometrical structure, electrical properties, and optical properties of Agdoped $\mathrm{ZnO}(0001)$ surface are systematically studied by first principles calculations.

\section{Computational methods}

All calculations are performed by the first-principles method based on the density functional theory with the generalized gradient approximation (GGA)[15], and the projector augmented wave (PAW)[16]pseudo-potentials, as implemented in the VASP codes[17, 18].

\footnotetext{
*Corresponding author: xiangqian8702@163.com
} 
The gamma centred $8 \times 8 \times 1 \mathrm{k}$-points is used for the Brillouin zone. The computational model contains five bilayers in which the two bottom $\mathrm{ZnO}$ bilayers are fixed while the top three $\mathrm{ZnO}$ bilayers and adatoms are allowed to relax. We carry out calculation with $10 \AA$ thick vacuum layers. To prevent unphysical charge transfer between the bottom and top slabs, pseudohydrogens are used on the bottom layer[19]. $\mathrm{Zn}^{3} \mathrm{~d}^{10} 4 \mathrm{~s}^{2}, \mathrm{O} 2 \mathrm{~s}^{2} 2 \mathrm{p}^{4}$ and $\mathrm{Ag} 4 \mathrm{~d}^{10} 5 \mathrm{~s}^{1}$ are chosen as valence electrons. The computational model is shown in Fig.1.

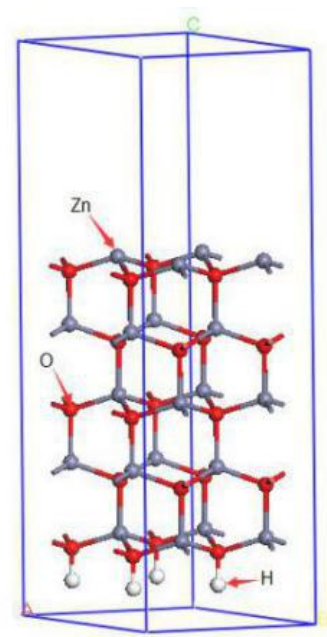

Fig. 1. Configurations of $\mathrm{ZnO}(0001)$ surface. Four pseudohydrogen atoms (white ball) are attached to the bottom of the cell

\section{Results and discussion}

\subsection{Geometry structure.}

By optimizing the geometry structure of the pure $\mathrm{ZnO}(0001)$ surface, The $\mathrm{Zn}-\mathrm{O}$ bilayer spacings are $0.493 \AA, 0.591 \AA$ and $0.601 \AA$ for the first three pairs (counted from the surface), which are slightly smaller than the value in bulk(0.634 $\AA$ ). On the other hand, The $\mathrm{Zn}-\mathrm{Zn}$ bilayer spacings are $2.711 \AA, 2.678 \AA, 2.663 \AA$ for the first three pairs (counted from the surface), which are slightly bigger than the value in $\operatorname{bulk}(2.638 \AA)$. This result shows that relaxation only affects the bilayer near the surface, but the relaxation of the deep bilayer is negligible. This result agrees with previous study[20].

\subsection{Electrical properties.}

The total density of states and projected density of states of Ag-doped $\mathrm{ZnO}$ (0001) surface at three layers are presented in Fig.2, respectively. From Fig. 2. We find that the Fermi levels are already in the conduction band, and the Fermi energy gradually moves into the deep conduction band as the doping layer deepened. The surface states near the conduction band minimum(CBM) are mainly contributed by the first layer of the $\mathrm{Zn}-\mathrm{O}$ bilayer, and the contribution of the second and third layer $\mathrm{Zn}-\mathrm{O}$ bilayers is less. Therefore, we chose projected density of states (PDOS) of Ag-doped $\mathrm{ZnO}(0001)$ surface at first layers to make further analysis. As shown in Fig.2.(b), The valence band maximum(VBM) of Ag-doped 
$\mathrm{ZnO}(0001)$ surface at first layers is mainly contributed by $\mathrm{Ag}-4 \mathrm{~d}$ and $\mathrm{O}-2 \mathrm{p}$, and the conduction band minimum is mainly contributed by $\mathrm{Zn}-4 \mathrm{~s}$ and $\mathrm{Ag}-5 \mathrm{~s}$.

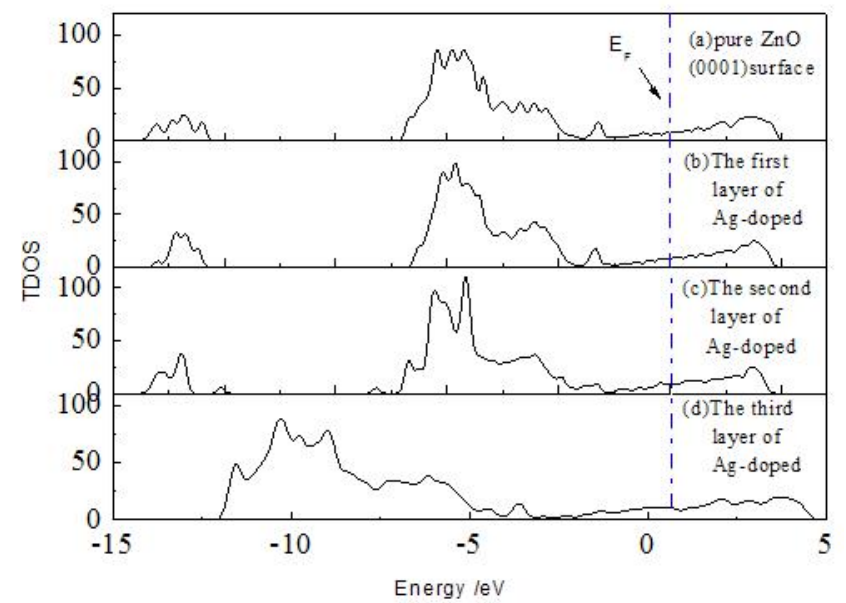

(a)

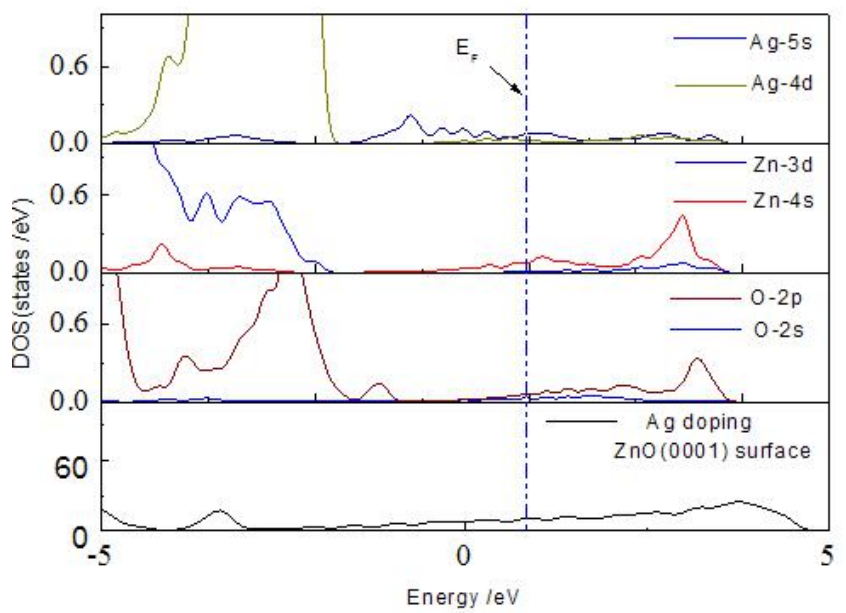

(b)

Fig. 2. (a): the total density of states (TDOS) of pure $\mathrm{ZnO}$ (0001) surface and the total density of states (TDOS) of Ag-doped $\mathrm{ZnO}(0001)$ surface. (b): projected density of states (PDOS) of Ag-doped $\mathrm{ZnO}(0001)$ surface at first layers.

In order to study the stability of doped surfaces, the formation energies and electrostatic potential of Ag doped with three layers on $\mathrm{ZnO}(0001)$ surface were calculated respectively. The formation energies are as follows: the first layer is $-1.65 \mathrm{ev}$; the second layer is $-1.39 \mathrm{ev}$; the third layer is $-1.29 \mathrm{ev}$. From the change of formation energy, it can be seen that the formation energy of $\mathrm{Ag}$ atom doped at the third layer position is highest, and the formation energy decreases gradually as the doping atom position moves to the surface. Therefore, we believe that $\mathrm{Ag}$ atoms are more likely to be doped on the first layer of $\mathrm{ZnO}(0001)$ surface.

In addition, we calculated the electrostatic potential energy of the pure $\mathrm{ZnO}$ (0001) surface and the electrostatic potential energy of the first layer doped Ag on the $\mathrm{ZnO}(0001)$ surface along the vertical surface ( $Z$ axis), as shown in Fig. 3. The electrostatic potential energy is obtained by the static calculation of the surface model after relaxation. In the calculated surface model, the work function is calculated from the electrostatic potential energy and Fermi energy. The work function is calculated as follows: 


$$
\phi=x-\varepsilon_{F}
$$

where $\phi$ is work function. $x$ is electrostatic potential energy. $\varepsilon_{F}$ is Fermi energy.

From the diagram, it is found that the electrostatic potential of each layer decreases as gradually enters the surface, especially at the first layer of the surface. The electrostatic potential fluctuates greatly, and the electrostatic potential energy is lower at the position near the center of the atom, and between the two atomic layers the value is the maximum. Especially after the first layer on the Ag doped $\mathrm{ZnO}$ (0001) surface, the electrostatic potential energy increases obviously. The main reason is that the surface structure changes greatly after surface relaxation. Through calculation, we can get that the work function of $\mathrm{ZnO}(0001)$ surface is $2.212 \mathrm{ev}$, and the experimental value is $2.8 \mathrm{ev}[21]$.

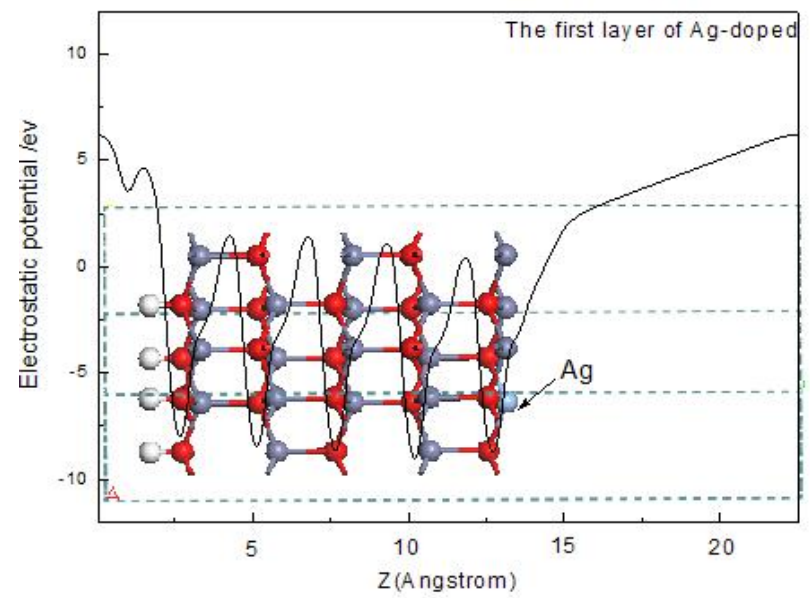

(a)

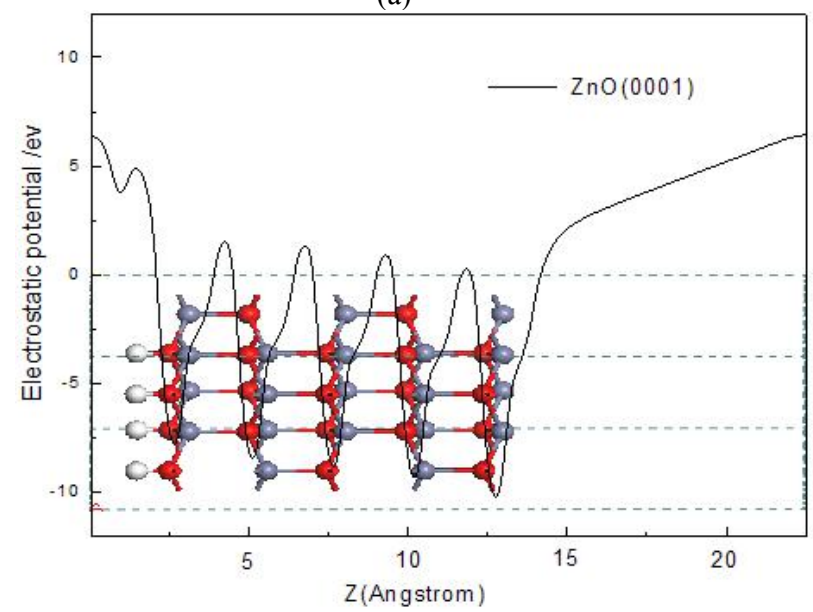

(b)

Fig. 3. (a)the electrostatic potential energy of the first layer doped $\mathrm{Ag}$ on the $\mathrm{ZnO}(0001)$ surface, (b)The electrostatic potential energy of the pure $\mathrm{ZnO}(0001)$ surface

\subsection{Optical properties.}

Sequentially, in order to reveal the relationship between the electronic structure and optical properties, it is necessary to study the imaginary part of the dielectric function. Fig.4 shows the dielectric function of pure $\mathrm{ZnO}(0001)$ surface and the first layer doped $\mathrm{Ag}$ on the $\mathrm{ZnO}$ 
(0001) surface. As we can see from the diagram, the imaginary part of their dielectric function is basically the same. However, the peak value is higher compared to the pure surface at $1.25 \mathrm{eV}$. Since each dielectric function peak corresponds to an absorption maximum, the corresponding absorption photon energy at the $1.25 \mathrm{eVenergy}$ is $4.23 \mathrm{eV}$. This is mainly due to the electronic transitions between the two orbital levels of Ag- $4 \mathrm{~d}$ and $\mathrm{O}-2 \mathrm{p}$.

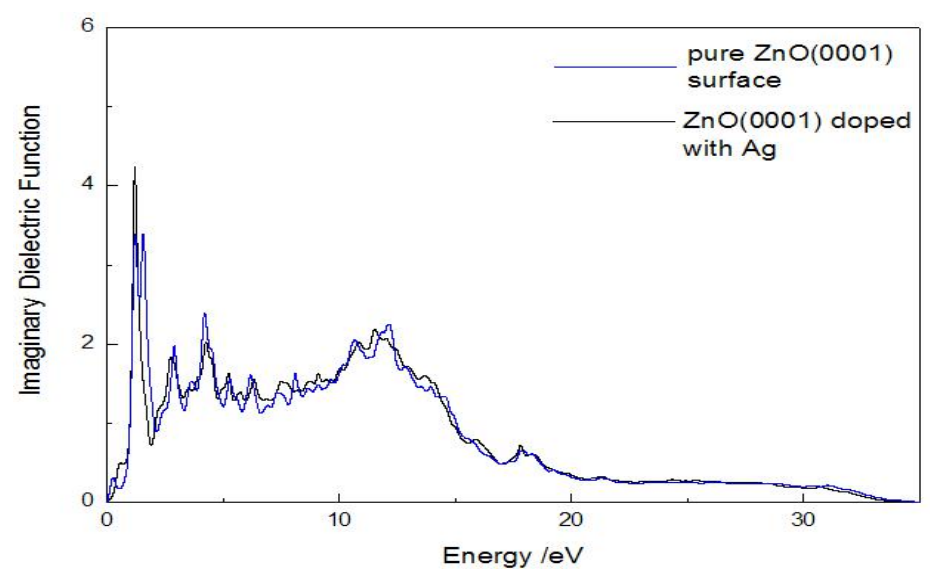

Fig. 4. the dielectric function of pure $\mathrm{ZnO}(0001)$ surface and the first layer doped $\mathrm{Ag}$ on the $\mathrm{ZnO}$ (0001) surface

\section{Conclusions}

In summary, geometrical structure, electrical properties, and optical properties of Ag-doped $\mathrm{ZnO}(0001)$ surface are investigated through the first-principle calculations. The calculated results show that the Fermi energy has entered into the conduction band after doping Ag, and the Fermi energy gradually move into the deep conduction band as the doping layer deepened. At the same time, we found that Ag preferred doping on the first layer of $\mathrm{ZnO}$ (0001) surface. From the overall point of view, the doped surface has gradually become an equipotential, showing obvious metallic characteristics. Simultaneity, A new peak is observed at $1.25 \mathrm{eV}$, which is mainly due to the electronic transitions between the two orbital levels of Ag- $4 \mathrm{~d}$ and $\mathrm{O}-2 \mathrm{p}$. We hope these results could provide useful information about metal atoms doped on $\mathrm{ZnO}$ surfaces.

This work was supported by Natural Science Foundation of Fuyang Teachers College (2016FSKJ12), National Natural Science Foundation of China(11604052), Natural Science Foundation of Anhui Province(KJ2017A343), Open subject of State Key Laboratory of Low-Dimensional Quantum Physics(KF201708).

\section{References}

1. D. C. Look, B. Claflin, Y. I. Alivov, S. J. Park, Physica Status. Solidi(a).201(2004).

2. K.Mahmood, B.S. Swain, Gill-Sang. Han, ACS Appl. Mater. Interfaces, 6(2014).

3. P.S. Venkatesh, K. Jeganathan, J. Solid State Chem, 200(2013).

4. Q. Li, L.P. Zhu, Y.G. Li, Journal of Alloys and Compounds, 697(2017).

5. M.A. Lahmer, Journal of Physics and Chemistry of Solids, 89(2016). 
6. D.M. Ma, Z. Wang, H.T. Cui, Sensors and Actuators B, 224(2016).

7. H. Xu, K. Ohtani, M. Yamao, H. Ohno, Appl. Phys. Lett, 89(2006).

8. R. Zhu, Q. Zhao, J. Xu, B.G. Liu, Cryst. Eng. Comm., 14(2012).

9. Z.L. Wang, X.Y. Kong, J.M. Zuo, Phys. Rev. Lett., 91(2003).

10. S. Pat, R. Mohammadigharehbagh, S. Özen, Vacuum, 141(2017).

11. L.X. Su, Y. Zhu, Q.L. Zhang, Applied Surface Science, 274(2013).

12. J.P. Xiao, T. Frauenhei, J. Phys. Chem. Lett, 3(2012).

13. H.F. Zhang, S.X. Lu, W.G. Xu, F. Yuan, Surface Science, 625 (2014).

14. M.A. Lahmer, K. Guergouri, Surface Science, 633(2015).

15. J.P. Perdew, Y. Wang, Phys. Rev. B, 45(1992) .

16. P. E. Blöchl, Phys. Rev. B, 50(1993).

17. G. Kresse, J. Hafner, Phys. Rev. B, 47(1993).

18. G. Kresse, J. Furthermuller,Phys. Rev. B, 54(1996).

19. X.Q. Dai, H.J. Yan, J.L.Wang, J. Phys: Condens. Matter, 20(2008).

20. Z. Yang, S.J. Xiong, Surface Science, 605 (2011).

21. X. Y, O.T. Hofmann, R. Patrick, B. Am. Math. Soc, 57(2012). 